


\title{
L'art de vivre en montagne, selon Charlotte Perriand
}

\author{
The art of mountain living, according to Charlotte Perriand
}

\begin{abstract}
Passionate about skiing and alpinism, the architect and designer Charlotte Perriand (1903-1999) developed numerous projects for loisirs and the mountain landscape over the course of her career. Her major projects in the years 1960 to 1980, such as her chalet in Méribel and the ski resorts of Les Arcs 1600 and 1800, owe their foundations to the research that she carried out in the 1930s.

The designs of this pioneer illustrate a way of living in harmony with nature and demonstrate how advanced her thinking was. Recognized as remarkable contemporary architecture, her designs remain extremely comfortable to live in today.
\end{abstract}

\section{Claire Grangé}

Holder of a DEA (Diploma of Advanced Studies) in History, and cultural media. She was named director of the museum "House of the Winter Olympics" from 1992 to 2018 where she was responsible for the development of exhibitions on subjects linked to development in the mountains, the architecture of ski resorts and winter sports. From 2007-2008, she oversaw the exhibition Charlotte Perriand and the mountain. In 2016, she wrote the book Charlotte Perriand, creating in the mountains, in collaboration with Guy Rey-Millet and Gaston Regairaz. She lives in the resort of Les Arcs (Savoie), which she has enjoyed since Christmas 1968, and where she is a ski instructor.
Keywords

Charlotte Perriand, modern architecture, design, ski resorts, mountain landscape. 


\author{
«L'homme et l'univers sont intimement liés, \\ c'est pourquoi je ne peux jamais séparer \\ les parties du tout pour ce qui concerne ma discipline, \\ l'architecture du milieu, l'équipement de \\ l'architecture. [...] \\ L'architecture procède de l'intérieur vers l'extérieur, \\ c'est un aller-retour».
}

Charlotte Perriand, Une vie de création, Odile Jacob, Paris, 1998, p. 29.

Passionnée de ski et d'alpinisme, l'architecte et designer Charlotte Perriand (1903-1999), a élaboré au cours de sa vie, de nombreux projets pour les loisirs et la montagne. Ses constructions maitresses des années 1960 à 1980, telles que son chalet de Méribel et les stations de ski des Arcs 1600 et 1800 trouvent leurs fondements dans les recherches qu'elle avait menées dans les années 1930 .

Les créations de cette pionnière illustrent un art de vivre en relation avec la nature et témoignent de la modernité de sa pensée. Reconnues en tant qu'Architecture contemporaine remarquable, ses réalisations sont toujours très agréables à habiter aujourd'hui.

\title{
Les recherches pour un habitat nouveau
}

A l'âge de 24 ans, Charlotte Perriand, jeune diplômée de l'Union centrale des Arts décoratifs, crée « Le bar sous le toit » qui est exposé au Salon des artistes décorateurs de 1927. Elle est immédiatement remarquée par la critique et intègre la même année l'atelier d'architecture de Le Corbusier et Pierre Jeanneret, rue de Sèvres (Paris 6 ${ }^{\mathrm{e}}$ ) comme associée pour l'équipement intérieur (1927-1937) et étudiante en architecture (1927-1934).

Dès cette période, elle pratique la haute montagne, avec son premier mari puis avec ses amis de l'atelier de la rue de Sèvres. Avec Pierre Jeanneret son compagnon de cœur de 1933 à 1940, ils vont parcourir, à pied et à ski, été et hiver, toutes les montagnes des Alpes. Au cours de ses excursions, Charlotte Perriand, sportive avant l'heure, intègre les leçons de

Image d'ouverture Chalet à Méribel, façade sud. Toutes les photographies: Pernette

Perriand-Barsac. vie de la montagne. Elle observe l'habitat et le mobilier des paysans, elle fait des croquis et des relevés de l'architecture vernaculaire.

Pendant les années passées à l'atelier de Le Corbusier, elle travaille certes sur la création des mo- biliers et les trois associés Le Corbusier-Jeanneret-Perriand déposeront, notamment, le brevet de la Chaise longue basculante (1928). Mais elle travaille aussi sur des projets d'aménagement tels que le projet Travail et Sport (1927) qui montre une pièce lumineuse aménagée comme un loft, avec un bar-cuisine ouvert, un bloc sanitaire couchage, un espace de sport et de gymnastique séparé par une cloison composée de volumes de rangements et, pour la détente, une mezzanine avec jardin suspendu et hamac (publié dans Répertoire du goût moderne, 1929).

Avec le stand de l'« Equipement intérieur d'une habitation, Le Corbusier, Pierre Jeanneret, Charlotte Perriand » présenté au salon d'automne de 1929 à Paris, qui illustre l'habitation idéale de "l'homme nouveau », les trois associés proposent une approche nouvelle de l'habitat basée sur la standardisation, la production d'équipements en série et sur la notion d'habitat minimum. Pour Le Corbusier, homme de formules qui marquent les esprits, l'équipement mobilier de « la machine à habiter » se résume à « des casiers, des chaises, des tables". A cause de la crise du logement et de l'insalubrité qui sévissent alors en Europe, la question de l'habitation minimum est une préoccupation des architectes modernes et figure à l'ordre du jour des CIAM de 1929 (Congrès internationaux d'architecture moderne). A l'occasion d'une étude approfondie sur l'architecture intérieure pour le logement ouvrier, Charlotte Perriand conclut que la surface minimum pour vivre décemment doit être de $14 \mathrm{~m}^{2}$ par habitant. Elle fait des propositions de logements pour célibataire, couple et familles de un à quatorze enfants, en rationalisant l'espace et en proposant une nouvelle organisation qui rompt totalement avec le modèle bourgeois : cuisines ouvertes, espaces modulables séparés par des cloisons coulissantes, rangements fonctionnels produits en série.

Charlotte Perriand, en véritable scientifique, étudie les gestes de la vie quotidienne, analyse les hauteurs accessibles pour une femme et définit une ergonomie du rangement qui libère l'espace.

Dans tous les aménagements qu'elle va concevoir durant sa vie, on retrouve cette « même philosophie 
du rangement de de l'utilisation de l'espace » (Barsac, 2005) qu'elle applique en particulier à ses réalisations en montagne, les refuges ou les appartements des Arcs.

\section{L'architecture pour les loisirs}

Le contexte des années 1930 est celui du développement des théories hygiénistes, des loisirs pour tous, de l'épanouissement grâce aux activités de plein-air.

Fig. 1 En juin 1936, le gouvernement du Front populaire Travail et sport, dessin de Charlotte

Perriand, 1927,

perspective

d'ensemble, planche

19, publié dans

Répertoire du goût

moderne, 1929

Pour toutes les

reproductions

d'œuvres: Charlotte

Perriand (c) ADAGP,

2019, Paris;

avec l'aimable

autorisation des

Archives Charlotte

Perriand. instaure deux semaines de congés payés pour tous les travailleurs. L'organisation des loisirs devient alors un centre d'intérêt.

Charlotte Perriand, qui a terminé sa formation et peut exercer comme architecte, participe aux concours lancés par la revue «L'Architecture d'aujourd'hui ». Dans le domaine des loisirs, elle préconise une architecture légère et préfabriquée, accessible au plus grand nombre. La simplicité de son projet de la Maison au bord de l'eau sur pilotis (1934) en est une belle illustration, dont elle dit: " Ce n'était pas une architecture conventionnelle de résidence secondaire, mais un espace éphémère plein de charme, intégré à la nature environnante ».
Sa réflexion s'appuie toujours sur la relation privilégiée avec la nature.

En 1935, elle conçoit le projet d'un « Hôtel de haute montagne à $2200 \mathrm{~m}$ d'altitude » où elle préconise d'examiner soigneusement l'emplacement : orientation, terrain, degré de pente, ensoleillement. La couverture en toits plats permet la récupération des eaux vers l'intérieur du bâtiment par des conduites à l'abri du gel et évite les chutes de neige sur les vacanciers. Les toitures sont recouvertes de gazon, ou de neige en hiver, pour servir d'isolation. L'été, les toits se transforment en solariums. Elle note que les calmes lignes horizontales de la toiture contrastent avec les formes découpées du paysage de la montagne. Elle imagine que le bâtiment suive la ligne de pente, ce qui dégage à l'aval différents étages alors qu'à l'amont il rentre de plain-pied dans le terrain.

Durant l'hiver 1938-1939, Pierre Jeanneret et Charlotte Perriand se rendent à Vars (HautesAlpes) et étudient un projet d'implantation de station de ski. Ils choisissent de proposer des résidences collectives, assez basses, qui descendent en cascade dans la pente.

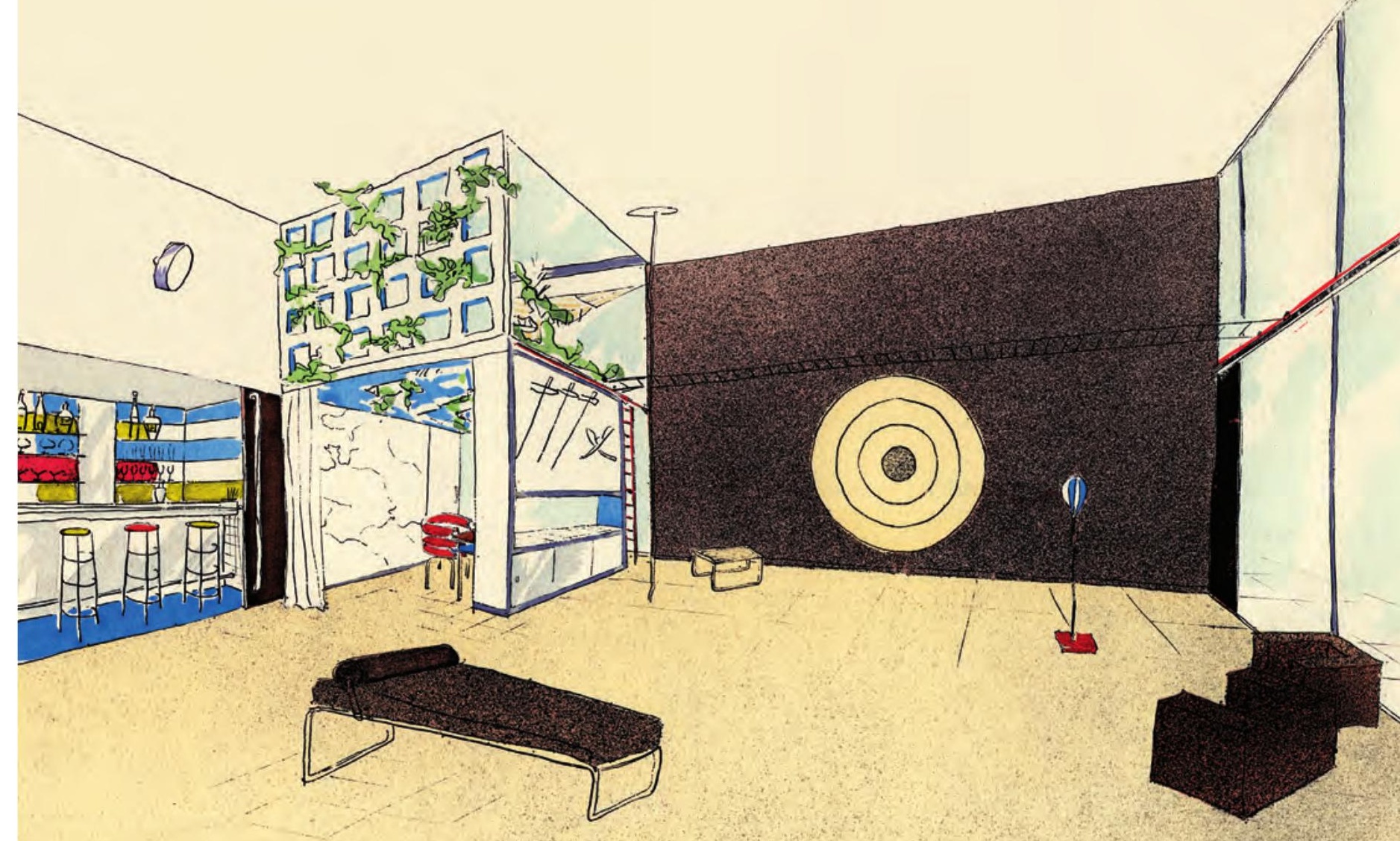



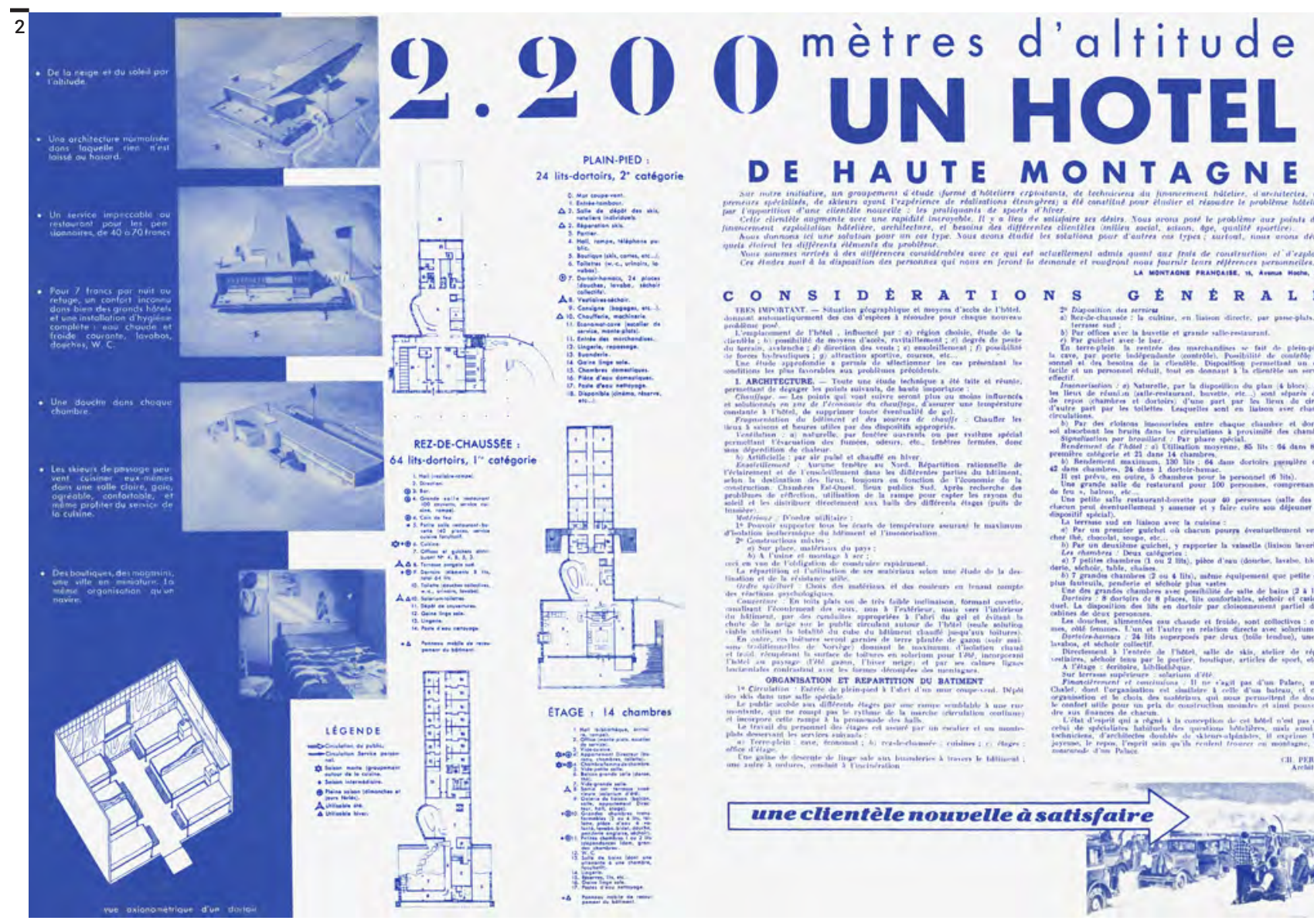

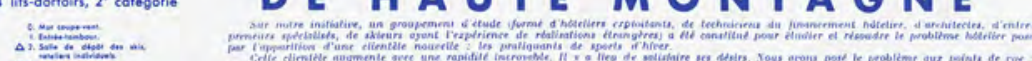

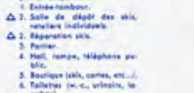

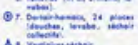
A. $\lim _{1} \cos x$

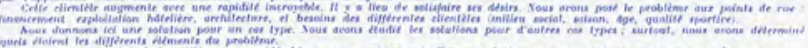

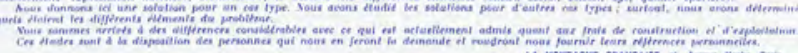

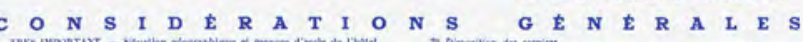

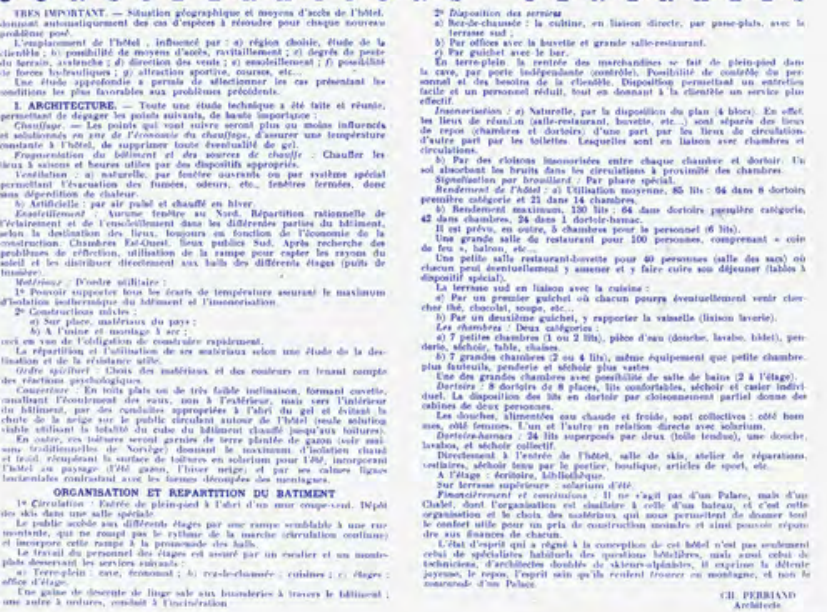
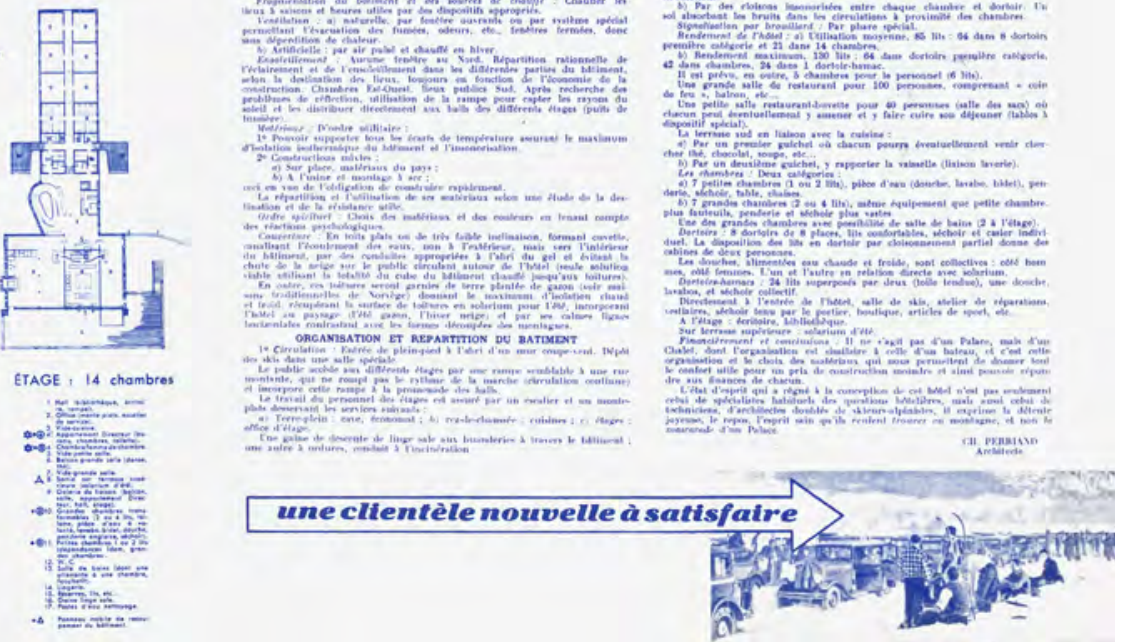

La station des Arcs où elle travaillera trente ans plus tard reprendra ces principes constructifs.

Montagnarde, sportive, Charlotte Perriand travaille également sur des projets de refuges. A une époque où les hélitreuillages n'existaient pas, elle s'attache à construire avec des matériaux faciles à transporter, légers et néanmoins isolants du froid. La structure est constituée par une ossature porteuse, tubulaire et métallique du type d'un échafaudage. Réalisé en 1937, avec l'ingénieur André Tournon, le refuge Bivouac est installé sur l'épaule du mont Joly (Haute-Savoie). En 1938, elle conçoit avec Pierre Jeanneret un autre refuge, resté à l'état de

Fig. 2

Projet d'un hôtel de haute montagne, 2200 m d'altitude, projet de Charlotte

Perriand, publié dans la revue Ski, 1935.

Fig. 3 Coupe et plan du refuge Bivouac, 1937, dessin de

Charlotte Perriand, André Tournon, ingénieur. maquette, de forme circulaire, évoquant une capsule spatiale. Le refuge Tonneau est constitué d'éléments préfabriqués et s'assemble comme un mécano en quelques jours. Sa forme offre peu de prise au vent et évite les accumulations de neige. Ce sont des constructions minimalistes qui répondent à la fonction d'abri exigée par les alpinistes. Charlotte Perriand étudie avec grand soin l'agencement intérieur de ces petits espaces où l'équipement, compact et transformable, doit répondre à une fonction. Les couchages superposés peuvent former banquette dans la journée, les matelas en trois parties s'empilent comme des poufs, la table abattante ferme le vaisselier, les tabourets sont aussi des coffres de rangement pour les affaires. Tous les usages sont calculés avec ingéniosité et le mouvement reste possible.

\section{Le Ghalet de Méribel}

En juin 1940, Charlotte Perriand est invitée pour un an au Japon comme conseillère pour l'art industriel. Elle y restera jusqu'en 1942, contrainte et forcée. Elle parcourt le pays, observe et découvre une civilisation avec laquelle elle se sent en harmonie et qui présente des similitudes avec l'esprit moderne : le plan libre, les façades ouvertes sur la nature, la standardisation des éléments modulables, la flexibilité des espaces, les cloisons coulissantes, les rangements incorporés à l'architecture. Elle écrit dans le numéro de mai 1956 de l'Architecture d'aujourd'hui : « La maison traditionnelle japonaise est le contraire de la nôtre. Elle procède de l'intérieur vers l'extérieur, l'horizon est le prolongement de la nature, la nature de la maison. Tout est mis en œuvre pour créer la détente par l'évasion. Cette évasion commence déjà sur le tatami, à l'intérieur, le regard plongeant sur l'infini ». Charlotte Perriand a trouvé au Japon une expression architecturale qui correspond à la relation qu'elle a toujours entretenue avec la nature. 

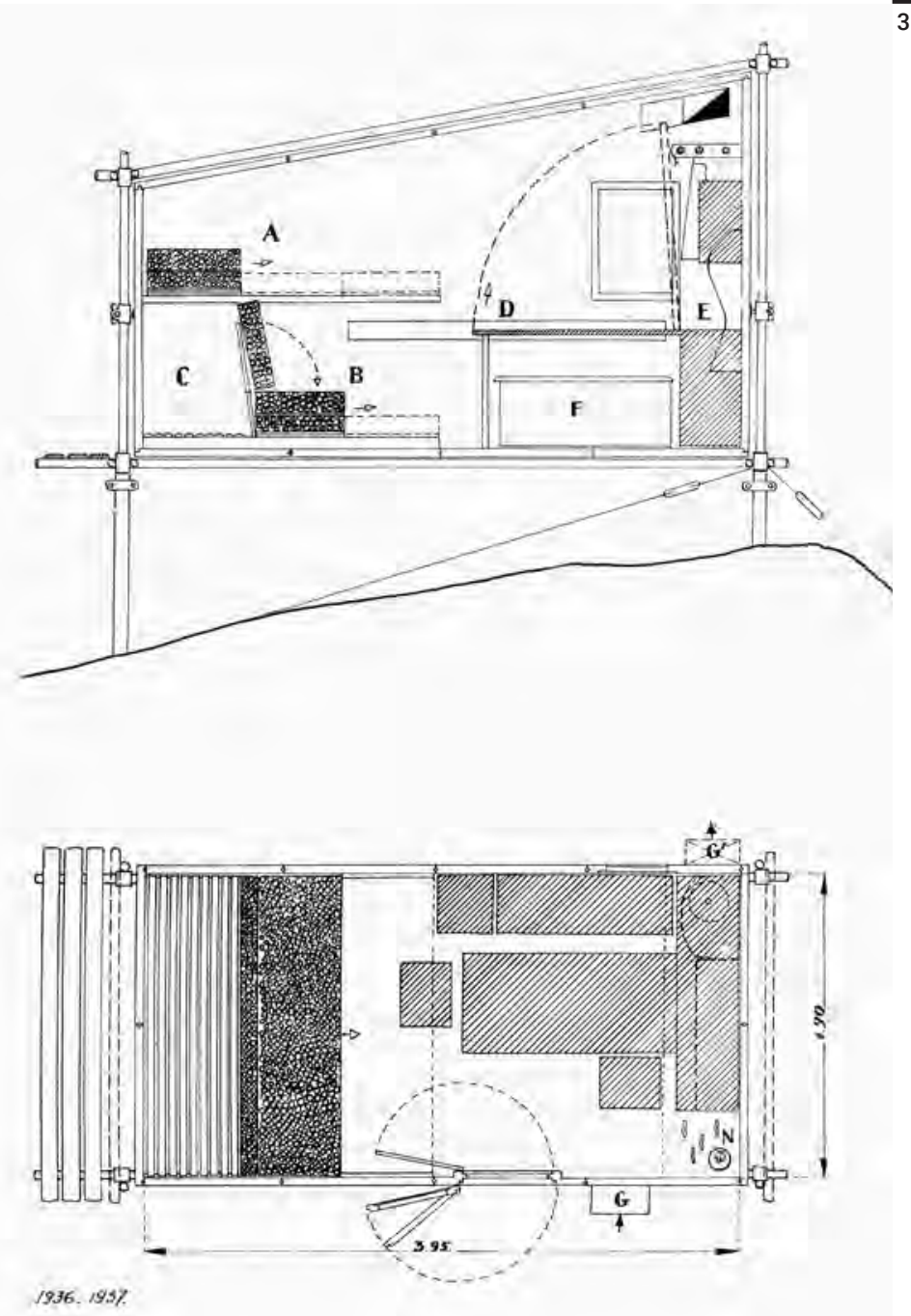

Figg. 4-5

Chalet à Méribel,

1960 - Grande pièce de vie à l'étage

- Architecte et

architecture intérieure

Charlotte Perriand.

Fig. 6

Façade sud.

Fig. 7

Charlotte Perriand à Méribel, 1987. des toitures à double pente. communication avec le paysage. quels est posée la charpente en bois, « comme un chapeau ", dit-elle. A l'étage, qui était réservé au grenier à foin, elle fait une seule pièce d'habitation et installe des grands pans de verre en double vitrage, ce qui ouvre l'espace intérieur vers l'extérieur, vers le soleil. Une simple terrasse sans balustrade est posée devant la baie vitrée pour favoriser encore la communication avec le paysage.

La grande pièce de l'étage ne comporte pas de séparation. Seules quelques cloisons coulissantes referment les alcôves des lits, comme les lits clos des maisons paysannes. Un déshabilloir précède l'espace affecté aux soins de toilette. La lumière zénithale pénètre par les pignons vitrés. Des panneaux coulissants translucides, qui s'escamotent dans les murs, filtrent la lumière. Enfin, autre clin d'œil aux dispositifs de l'architecture japonaise, le sol en paille de riz qui possède la douceur du tatami.

Au rez-de-chaussée, la pièce à vivre est organisée à partir d'une grande cheminée ouverte en granit, entourée de banquettes, dans laquelle on entre pour se réchauffer, faire des grillades, chanter, lire... Devant la baie vitrée qui descend jusqu'au sol, elle a disposé une simple table en rondins, quelques tabourets tripodes et un fauteuil paillé pour inviter à contempler la prairie alpine et les traces d'animaux. Ce chalet, adossé à la pente avec légèreté, est en connivence avec le lieu et évoque la simplicité de l'abri des montagnards. D'une superficie totale d'environ $85 \mathrm{~m}^{2}$, il est une synthèse entre l'interprétation des sources traditionnelles et l'esprit créatif qui élabore un art d'habiter contemporain. C'est une expérimentation de la modernité en montagne, sans régionalisme, ni folklorisme. Charlotte Perriand parlait «d'une architecture franche » dont la qualité a été reconnue par l'attribution du classement monument historique en 2016.

De retour en France en 1946, après un séjour en Indochine, Charlotte Perriand travaille à l'aménagement de la jeune station de Méribel (Savoie). Le promoteur Peter Lindsay, qui ne peut la payer, lui offre un terrain qu'elle choisit au fil de ses pas, dans une jolie combe où coule un ruisseau. Ce n'est qu'en 1960 qu'elle décide de construire son chalet qu'elle conçoit comme un refuge. Son premier projet, dessiné en 1948-1949, avec des terrasses ouvrant sur la montagne et une grande baie vitrée pivotante formant coupe-vent, comporte une toiture à toit plat végétalisé. Ce projet est refusé car il ne correspond pas au cahier des charges de Méribel qui impose

Elle change de démarche et entreprend d'arpenter les villages de la vallée pour analyser les techniques de construction. Elle va alors réaliser un chalet au charme fou qui allie la simplicité du refuge à la

Des maisons traditionnelles savoyardes, elle conserve la structure faite de murs de pierre arasés sur les-

\section{Les stations des Arcs 1600 et 1800}

C'est avec la conception de la station de ski des Arcs 1600 et 1800 que Charlotte Perriand va pleinement exprimer son talent et restituer tout son savoir-faire. A l'âge de 64 ans, elle va s'engager sans réserve dans ce projet de grande ampleur et lui consacrera plus de vingt ans, de 1967 à 1989. Il s'agit de définir le programme d'une station de 30000 lits, répartis en trois entités. C'est une opération complexe, alliant l'urbanisme à l'architecture, sur un site vierge et en respectant des délais rigoureux de construction.

Charlotte Perriand conduit un travail collectif mené avec une équipe composée des architectes-urbanistes Gaston Regairaz et Guy Rey-Millet de l'AAM (Atelier d'Architecture en Montagne), de Bernard Taillefer, charpentier-menuisier qui deviendra architecte en 1982, de Pierre Faucheux, graphiste et architecte, d'Alain Tavès et Robert Rebutato, architectes et anciens de l'atelier de Le Cor- 

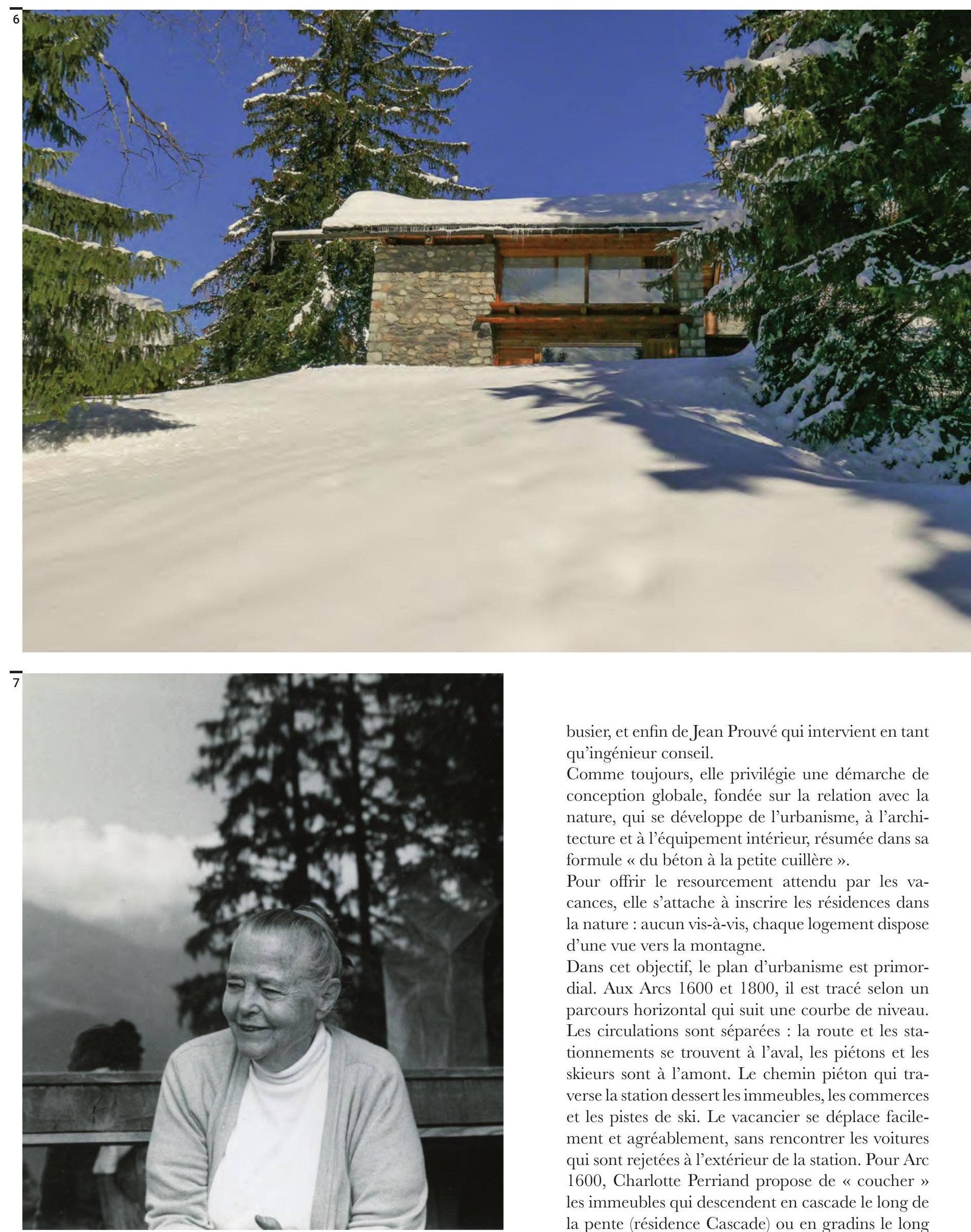

busier, et enfin de Jean Prouvé qui intervient en tant qu'ingénieur conseil.

Comme toujours, elle privilégie une démarche de conception globale, fondée sur la relation avec la nature, qui se développe de l'urbanisme, à l'architecture et à l'équipement intérieur, résumée dans sa formule « du béton à la petite cuillère ».

Pour offrir le resourcement attendu par les vacances, elle s'attache à inscrire les résidences dans la nature : aucun vis-à-vis, chaque logement dispose d'une vue vers la montagne.

Dans cet objectif, le plan d'urbanisme est primordial. Aux Arcs 1600 et 1800, il est tracé selon un parcours horizontal qui suit une courbe de niveau. Les circulations sont séparées : la route et les stationnements se trouvent à l'aval, les piétons et les skieurs sont à l'amont. Le chemin piéton qui traverse la station dessert les immeubles, les commerces et les pistes de ski. Le vacancier se déplace facilement et agréablement, sans rencontrer les voitures qui sont rejetées à l'extérieur de la station. Pour Arc 1600, Charlotte Perriand propose de « coucher» les immeubles qui descendent en cascade le long de la pente (résidence Cascade) ou en gradins le long 

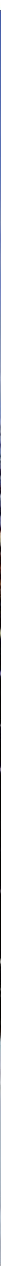

du plateau (résidence Cachette). Le résultat, qui évoque un jeu de dominos, est la juxtaposition de petites unités qui épousent le terrain et conservent la vue vers le massif du Mont-Blanc ou vers la vallée de la Tarentaise. Lorsqu'elle conçoit l'ensemble Versant Sud et Adret avec Gaston Regairaz, elle reprend les principes du concours pour l'aménagement de la vallée des Belleville (Savoie, 1962). Les

Fig. 8 immeubles sont placés en travers de la pente, encasVersant Sud, trés dans le terrain, en mono-orientation, face au intérieur d'un studio sud-ouest. Elle parle d'une « architecture bioclima- Architecture tique ouverte au soleil ». Ces dispositions créent un intérieure, cadre de vie ouvert vers la montagne, les sommets équipement et mobilier Charlotte Perriand.

Fig. 9

Versant Sud, Arc 1600,1971 Architectes Charlotte Perriand et Atelier d'architecture en montagne / Gaston

Regairaz et Guy

Rey-Millet. ou l'horizon et favorisent le délassement.

Pour Arc 1800, elle intervient différemment pour respecter le programme demandé d'une plus grande densification. Les constructions sont concentrées à des endroits déterminés ce qui permet de dégager entre elles des espaces de nature et de conserver les vues vers le paysage. Les bâtiments sont disposés en peigne, autour du plateau laissé libre pour installer les activités sportives, golf en été ou ski en hiver. Pour respecter l'ampleur du projet, s'inspirant des projets de l'hôtel de haute montagne (1935) et de Vars (1939), elle inscrit les bâtiments de grande hauteur dans le sens de la pente et garde une ligne de toiture horizontale. La différence créée entre le haut et le bas permet d'obtenir un immeuble de plusieurs étages côté aval. Alors qu'à l'amont, la toiture démarre de plain-pied depuis le chemin et devient même une circulation avec des commerces. Ainsi, dix-huit mille lits sont construits à Arc 1800, qui profitent d'une vision vers la montagne alors que l'espace de l'alpage est préservé.

Dans les aménagements intérieurs, Charlotte Perriand utilise ses recherches sur l'habitat minimum de 1929 et celles sur les refuges où elle a mis en pratique la polyvalence des lieux. Elle favorise le bienêtre du vacancier par la communication avec la montagne. Devant la grande baie vitrée coulissante, elle installe une banquette qui prolonge la terrasse à l'intérieur. Recouverte d'un matelas tripartite, c'est une place propice au repos ou aux bains de soleil. A Arc 1600, les terrasses des façades sud sont décalées en escalier, ce qui donne le même ensoleillement à tous les étages.

Pour les logements, elle définit une largeur minimum de façade de $3,66 \mathrm{~m}$, puis l'appartement se dé- 

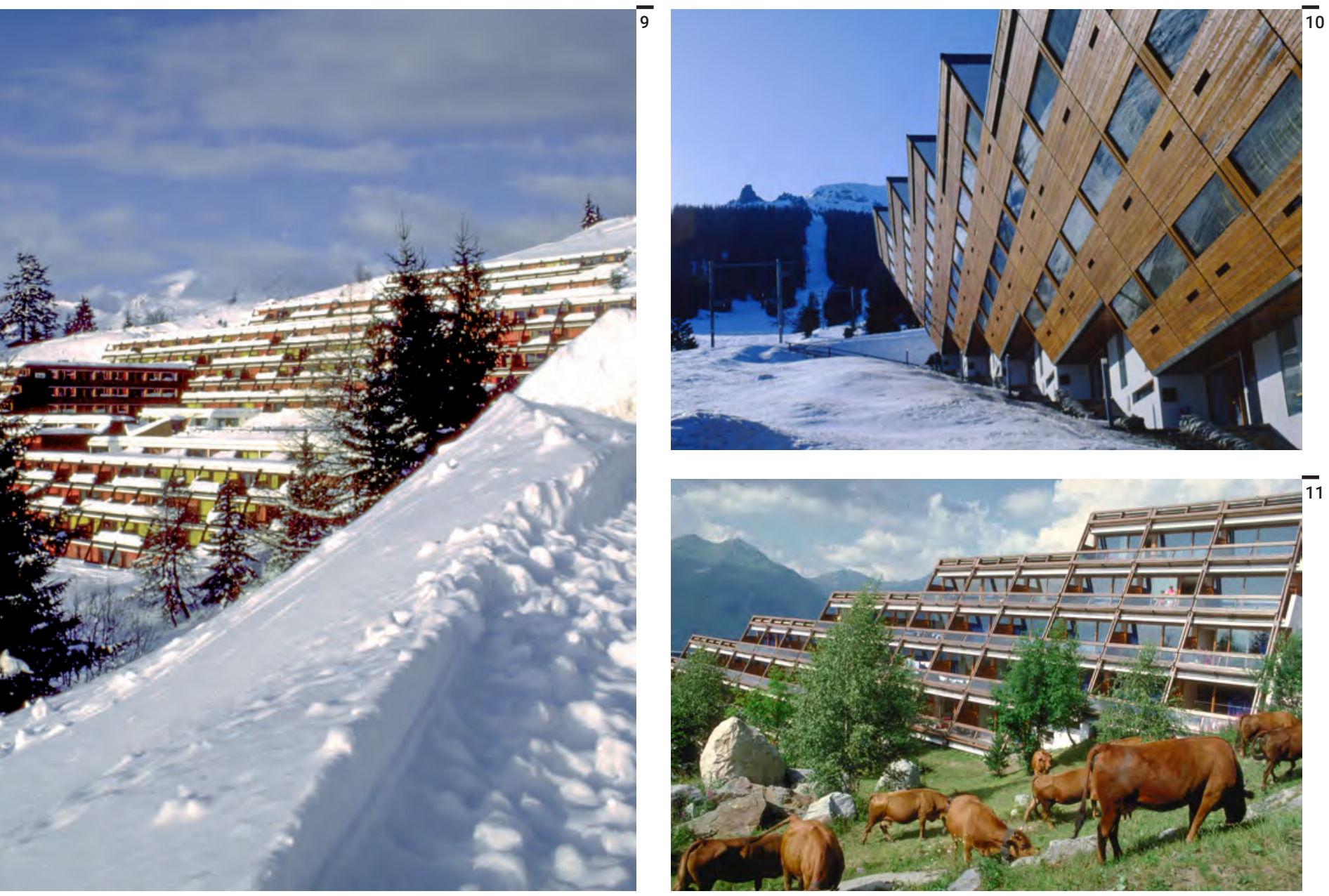

Figg. 10-11

La Cascade, Arc 1600, 1969 -

Façade nord-est et sud-ouest Architectes Charlotte Perriand et Atelier d'architecture en montagne / Gaston

Regairaz et Guy

Rey-Millet. ploie en profondeur. Le logement est conçu comme un seul volume avec un agencement spécifique et des rangements intégrés à l'architecture. « Sans un rangement bien conçu, pas de vide possible dans l'habitat » avait-elle écrit dans le numéro spécial de la revue «Techniques et Architecture » publié en août 1950 et intitulé L'Art d'habiter.

Elle étudie un seul emplacement pour les gaines techniques et arrivées d'eau, au milieu du logement, où elle installe la salle de bain, couplée aux toilettes et à la cuisine. A cause des délais de construction réduits à huit mois, elle imagine des salles de bain préfabriquées, à partir de 1975 pour les résidences Belles Challes et Lauzières (Arc 1800). Les cabines de bain, fabriquées en usine, composées de deux coques avec un joint horizontal comprenant tous les sanitaires, sont livrées en un bloc et installées à la grue au fur et à mesure de l'avancement des coffrages béton. Charlotte Perriand avait fréquemment observé que les interventions des différents corps de métier ralentissaient les chantiers.

Elle place la cuisine ouverte au centre de l'appartement, avec un simple bar de sapin dessiné en forme, pour délimiter visuellement les espaces. La cuisine est intégrée à la pièce de vie, la maîtresse de maison communique avec sa famille et elle dispose partout d'un lave-vaisselle pour faciliter les tâches ménagères en vacances.

Dans ces studios de $29 \mathrm{~m}^{2}$ habitables, en moyenne, elle veille aux proportions définies au Modulor. Dès l'entrée, elle calcule la longueur de deux lits superposés, équipe les murs de rangements. Elle garde un espace de vie ouvert vers le soleil, avec une table spécialement dessinée et des banquettes pouvant servir de lits.

A la demande du promoteur Roger Godino en 1984, elle étudie un modèle de studios-loisirs, souvent appelé les « chalets de Charlotte Perriand » pour le village de Charmettoger (Arc 1800). Elle conçoit ces duplex à l'image de refuges avec une mezzanine décalée, fixée par des fers IPN, où sont installés les couchages. Au rez-de-chaussée, elle agence avec précision les modules préfabriqués des sanitaires et de la kitchenette. Elle intègre des rangements sous les marches de l'escalier. La pièce de séjour, meublée d'une table, de tabourets et d'un divan dépliant, reste un volume ouvert qui profite de la double hauteur vitrée. 


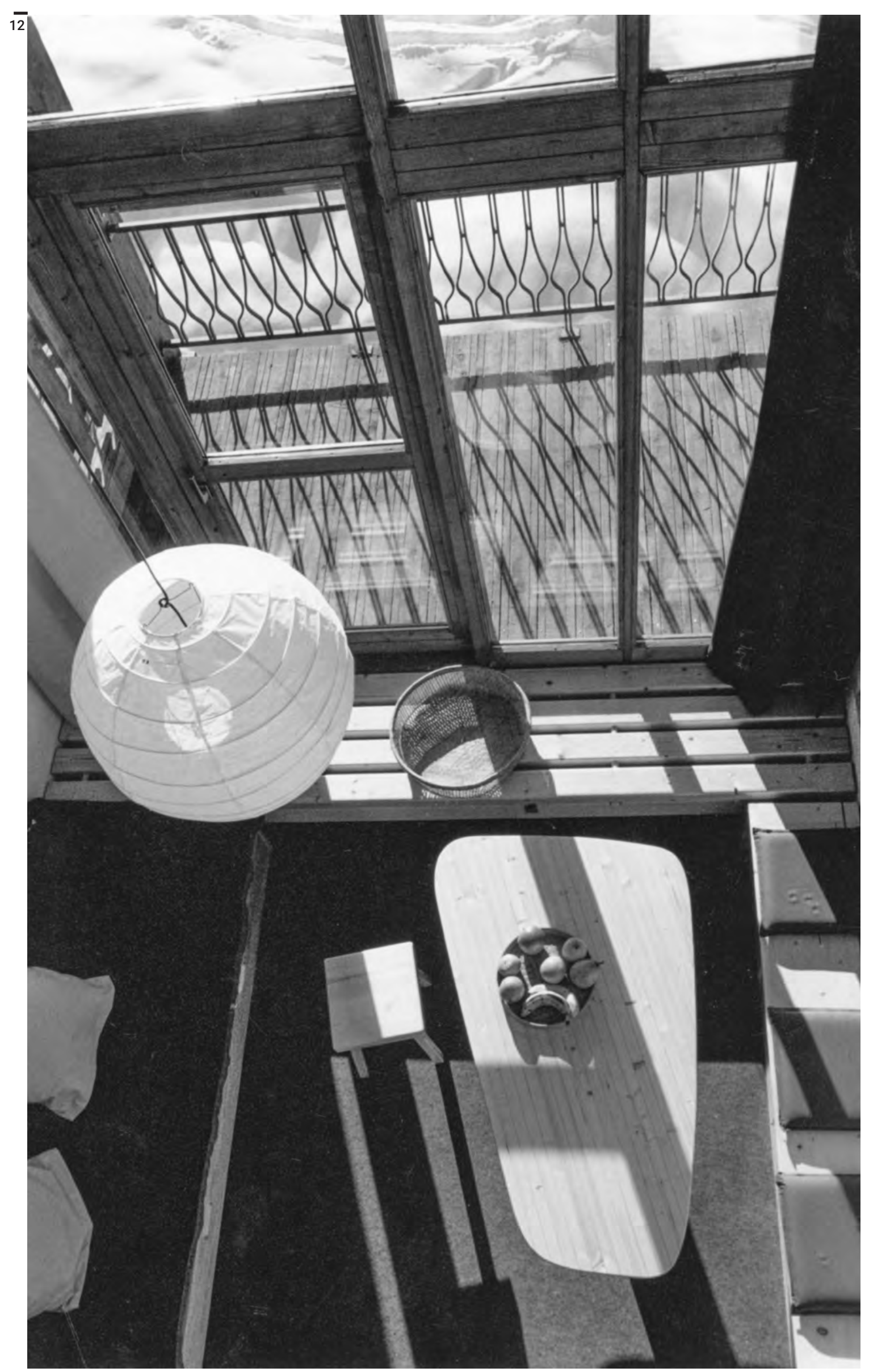




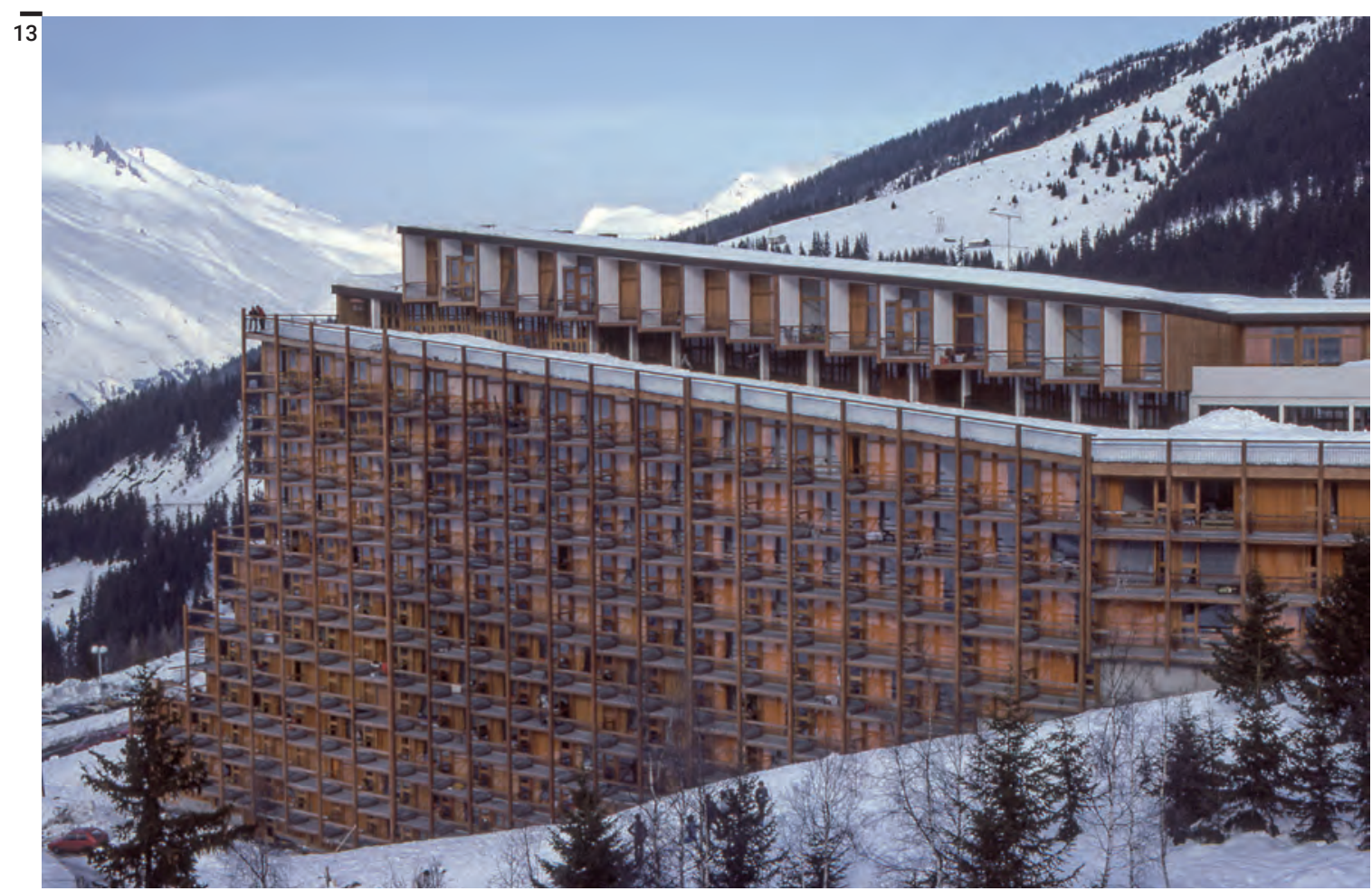

Fig. 13

Résidence Pierra Menta, Arc 1800 1976 - Architectes Charlotte Perriand, Bernard Taillefer, André Chedal / COGED, Roger Boulet.
Dans les aménagements que l'architecte Charlotte Perriand réalise aux Arcs, elle a travaillé avec ingéniosité, d'une façon économique et dans le souci d'offrir les loisirs au plus grand nombre. C'est en montagnarde qu'elle aborde la conception de l'habitat, en privilégiant le rapport essentiel avec la nature. C'est en femme et en sportive qu'elle conçoit les intérieurs en laissant place au mouvement, car selon Charlotte Perriand, l'habitat doit « créer les conditions de l'équilibre humain et de la libération de l'esprit ». Cette œuvre reste d'une grande modernité et a été reconnue Architecture contemporaine remarquable / Label XXe siècle en 2003.

Remerciements à Jacques Barsac pour sa relecture attentive et à Pernette Perriand-Barsac pour ses précieux conseils.

\section{Bibliographie}

Barsac Jacques (2005), Charlotte Perriand, Un art d'habiter (1903-1959), Norma, Paris.

Barsac Jacques (2008), Charlotte Perriand et le Japon, Norma, Paris.

Barsac Jacques (2015 à 2019), Charlotte Perriand, L'œuvre complète, vol. 1 (1903-1940), vol. 2 (1940-1955), vol. 3 (1956-1968), vol. 4 (1968-1999), Norma, Paris.

Cherruet Sébastien, Barsac Jacques (sous la direction) (2019), Le monde nouveau de Charlotte Perriand, Gallimard/Fondation Vuitton, Paris.

Godino Roger (1996), Construire l'imaginaire ou la quête inachevée d'un aménageur, éd. HID, Paris (épuisé).

Grangé Claire (sous la direction) (2016), Charlotte Perriand, Créer en montagne, éd. CAUE de Haute-Savoie, collection Portraits, Annecy.

Lyon-Caen Jean-François (sous la direction) (2003), Montagnes territoires d'inventions, École nationale d'architecture de Grenoble, Grenoble.

Lyon-Caen Jean-François (sous la direction) (2014), Stations de sports d'hiver, urbanisme et architecture, Région Auvergne-Rhône-Alpes, inventaire général du patrimoine culturel, Lieux Dits, Lyon.

Perriand Charlotte (1998), Une vie de création, Odile Jacob, Paris.

Perriand-Barsac Pernette (sous la direction) (2013), Charlotte Perriand, Carnet de montagne, éd. Maison des Jeux olympiques d'hiver, Albertville (épuisé). 